\title{
New psychotherapeutic approaches in adult ADHD - acknowledging biographical factors
}

\author{
Caroline Lücke ${ }^{1 *}$, Alexandra P. Lam' ${ }^{1}$, Helge H. O. Müller' ${ }^{1}$, Alexandra Philipsen ${ }^{1,2}$ \\ 'Medical Campus University of Oldenburg, School of Medicine and Health Sciences, Psychiatry and Psychotherapy - University Hospital, Karl-Jaspers-Klinik, \\ Bad Zwischenahn, Germany \\ ${ }^{2}$ Department of Psychiatry and Psychotherapy, Medical Faculty, University Medical Center - University of Freiburg, Freiburg, Germany
}

Article Info

Article Notes

Received: June 11, 2017

Accepted: July 10, 2017

\section{${ }^{*}$ Correspondence:}

Caroline Lücke, Medical Campus University of Oldenburg,

School of Medicine and Health Sciences, Psychiatry and

Psychotherapy - University Hospital, Karl-Jaspers-Klinik, Bad

Zwischenahn, Germany, Email: Caroline.Luecke@uol.de

(c) 2017 Lücke C. This article is distributed under the terms of the Creative Commons Attribution 4.0 International License

\section{Keywords}

Adult ADHD

Psychotherapy

Schema therapy

Early maladaptive schemas

Dysfunctional patterns

Pharmacological non-responder

\section{ABSTRACT}

Cognitive behavioral therapy (CBT) is the standard form of psychotherapy currently used in adult attention deficit hyperactivity disorder (ADHD). However, biographical factors, such as chronic negative feedback in childhood, which may likely play a role in ADHD as a developmental disorder, are usually not substantially addressed by CBT. In recent years, schema therapy has received increasing attention as an effective therapy approach for chronic psychiatric disorders. A core feature of schema therapy is the identification and targeting of early maladaptive schemas, which are dysfunctional patterns and beliefs resulting from childhood experiences. Recently, two studies have demonstrated an increased prevalence of maladaptive schemas in adult ADHD. Thus, schema therapy might constitute a potentially promising approach in the treatment of ADHD, especially with regard to secondary problems such as poor coping strategies or impaired self-perception. However, randomized controlled clinical studies are needed to support that theory. Here, we provide an overview on the topic of biography-oriented therapy approaches in relation to adult ADHD, summarize current literature and discuss implications for future research.

\section{Background: Current practice of psychotherapy in ADHD}

Attention deficit hyperactivity disorder (ADHD) is a chronic psychiatric disorder, starting in early childhood and often continuing into adult age. With its three main clinical features, inattention, hyperactivity and impaired impulse control, ADHD frequently interferes with the professional and private life of patients, leading to a variety of problems such as underachievement at work, disturbance of relationships and social contacts or even to serious secondary disorders like depression (review: ${ }^{1}$ ). According to the criteria in the Diagnostic and Statistical Manual of Mental Disorders, $5^{\text {th }}$ edition (DSM-5) for the diagnosis of ADHD, several symptoms of inattention and hyperactivity-impulsivity must be present, occur in at least two situations, interfere with daily functioning, and must have been present before the age of 12 years ${ }^{2}$. Pharmacological therapy with methylphenidate is regarded to be firstline treatment in adult ADHD and is very effective in alleviating ADHD core symptoms ${ }^{3}$. However, a considerable proportion of patients have residual symptoms under medication ${ }^{4}$ or have contraindications for medication. Therefore, alternatives to pharmacological approaches are needed. In the development of psychotherapeutic approaches, most programs have been based on the principle of cognitive behavioral therapy (CBT) $)^{5,6}$ and there is evidence that these are effective in reducing ADHD symptoms, at least with regard to self-reported symptom evaluation ${ }^{7}$. Furthermore, approaches that combine principles of CBT with aspects of dialectic 
behavior therapy (DBT) have been developed, offering ADHD patients further strategies to specifically target difficulties in emotion and impulse control, based on the concept of mindfulness ${ }^{8}$. A common feature of the existing psychotherapeutic treatments in ADHD is a main emphasis on the ADHD symptoms and their direct consequences in daily life, while internalized secondary problems, such as low self-esteem or a depressive coping-style are usually not the primary focus of these programs ${ }^{5}$.

General developments in psychotherapeutic programs: incorporation of biographical factors

In the development of new psychotherapeutic approaches on other psychiatric conditions, programs have been suggested, that, in addition to behavioral work, integrate the recognition and targeting of patients' (usually negative) biographical experiences. While, of course, the general idea of targeting childhood traumas is not new, and is an important factor in traditional psychoanalysis, the idea to combine and integrate this focus with behavioral-based approaches is a relatively young development. One of these promising approaches is Cognitive Behavioral Analysis System of Psychotherapy (CBASP) ${ }^{9}$, which is already widely used in clinical practice, however this program has been specifically developed for the treatment of chronic depression and, to our knowledge has not been adapted for use in other psychiatric conditions. Another important psychotherapeutic approach acknowledging a patient's personal history is Schema therapy, developed by Young et al. ${ }^{10}$. Schema therapy seeks to identify and alleviate early maladaptive schemas. These are internalized assumptions and expectancies an individual has developed about their own identity in relation to the people and the world around them. Early maladaptive schemas are formed during childhood from chronic negative experiences with others, such as parents or peers, interfere with the development of a stable and resilient personality, and persist until adulthood. Examples of early maladaptive schemas are "Abandonment/Instability", referring to the expectation that one will soon lose anyone with whom an emotional attachment has been formed, or "Failure", referring to the belief that one is incapable of performing as well as one's peers in different areas of life ${ }^{10}$. As described by Young, the identification of a patient's temperamental factors and their interaction with other developmental factors is part of the structured assessment process in schema therapy, since these factors may contribute to the development and manifestation of the patient's specific maladaptive schemas $^{10,11}$. Schema therapy seeks to reduce the power early memories and feelings linked to maladaptive schemas have on the patients' functioning and well-being until the present day, and tries to replace maladaptive behavior with more mature beliefs and strategies, such as the basic assumptions of self-worth and self-efficacy and the ability of appropriate problem-solving. Schema therapy is not restricted to the use in certain psychiatric conditions but was originally developed for patients with severe, chronic conditions, such as personality disorders, who do not sufficiently respond to usual psychotherapeutic treatment. The extension of schema therapy to the areas of depression and anxiety has been suggested, and is subject to current $\operatorname{research}^{12,13}$.

\section{Existence of maladaptive schemas in ADHD: results from recent studies}

Given that ADHD is a chronic developmental disorder, usually starting in early childhood, it seems reasonable to assume that biographical factors may play a role in the psychopathology of adult ADHD patients as well. Children with ADHD often show a poor school performance due to inattention, are reprimanded for hyperactive behavior or get into conflicts due to their impulsivity. The often noisy and disruptive behavior of ADHD patients promotes harsh and inappropriate reactions from parents and caregivers and many children with ADHD are rejected by their peers for violent behavior ${ }^{14}$. Transferred to Young's theory of schema therapy, ADHD traits could be seen as temperamental factors interplaying with other biographical circumstances in the development of maladaptive schemas. Already more than a decade ago, Ramsay \& Rostain discussed the importance of internalized core beliefs, also known as schemas, in relation to adult ADHD and ADHD-specific psychotherapy ${ }^{15}$ and suggested the targeting of a patient's "compensatory strategies", linked to his schemas, by CBT. The same authors created the concept of ADHD as an "Axis 1.5 disorder", emphasizing the fundamental influence of ADHD traits on a patient's personal development ${ }^{16}$.

Recently, two studies have systematically investigated the presence of early maladaptive schemas in adult ADHD: Miklosi et al. ${ }^{17}$ assessed a sample of non-clinical adults for symptoms of ADHD, using the Adult ADHD SelfReport Scale (ASRS) ${ }^{18}$, as well as for the manifestation of maladaptive schemas, using 4 subscales of the short form Young Schema Questionnaire (YSQ) ${ }^{10}$. Participants who showed elevated scores for more than 8 ADHD symptoms on the ASRS, suggesting a possible ADHD diagnosis, had significantly higher scores on the YSQ for all investigated subscales (social isolation, defectiveness/shame, failure, and insufficient self-control/self-discipline). While this study had some limitations, including the lack of a clinical, observer-based ADHD diagnosis in "patients", the small number of individuals with ADHD symptoms $(n=15)$, and the investigation of only a small proportion of maladaptive schemas, it provided first implications that maladaptive schemas may be increased in ADHD patients.

In a further study by our own group ${ }^{19}$ the existence of maladaptive schemas was investigated in a clinical sample 
of 80 adults with diagnosed ADHD and compared to a control group of 80 healthy adults. ADHD diagnoses were secured clinically by a careful assessment of the ICD10 and DSM-IV diagnostic criteria, including a retrospective evaluation of childhood ADHD using the Wender Utah Rating Scale ${ }^{20}$. Furthermore the CAARS questionnaire ${ }^{21}$ and the ADHD Checklist ${ }^{22}$ were used to examine the severity and the subtype of ADHD in patients. Participants were screened for the whole range of maladaptive schemas as defined by Young et al. ${ }^{10}$, using the complete YSQ-S2. ADHD patients had significantly higher YSQ scores compared to healthy controls for all maladaptive schemas, with the exception of "Vulnerability to Harm or Illness". In line with the expectations based on typical biographical experiences of ADHD patients, the schemas "Failure", "Defectiveness/ Shame", "Subjugation" and "Emotional Deprivation" were most pronounced in ADHD sufferers. The fact that the "Vulnerability to Harm or Illness" schema did not significantly differ between groups corresponds well with the increased prevalence of high-risk behavior in ADHD patients, such as tobacco or drug usage, fast driving or risky sexual behavior ${ }^{23}$. This tendency for high-risk behavior has been connected to an "intuitive-existential" style of decision making in $\mathrm{ADHD}^{24}$, with a primary focus on the expected reward of an action and an inclination to neglect the possibility of harmful events. It thus seems plausible to assume that this internal trait of ADHD counteracts negative external experiences, which could lead to development of a "Vulnerability" schema, resulting in normal levels of this schema in ADHD patients.

In addition to the existence of maladaptive schemas, the study also showed a significantly elevated prevalence of comorbid psychiatric disorders in ADHD patients compared to healthy controls. Particularly affective disorders, anxiety, alcohol abuse and personality disorders were common comorbidities in the ADHD group, all of which can be seen as potentially linked to maladaptive schemas.

On an individual level, Weusten et al. $^{25}$ have recently described an interesting case of schema therapy in an elderly patient with a severe personality disorder, dermatillomania and comorbid ADHD. With increasing age, the overall psychiatric burden of this patient had accumulated, especially after forced retirement, with the consequential loss of structure and social contacts. The authors discuss how the undiagnosed and untreated ADHD likely had profound influence on the development of the patient's personality disorder, which was connected to the maladaptive schemas "Failure", "Social alienation" and "Lack of self-control." Schema-focused therapy was conducted in this patient and an improvement regarding his most prominent schemas as well as his dermatillomania could be reached, while some of his symptoms, like anxiety, had increased after the first 20 therapy sessions. A concurrent medication with Methylphenidate, which may also have helped with his ability to profit from schema therapy, was declined by the patient. This case study underlines the fact that, while schema therapy seems an interesting therapy approach in ADHD, the interplay with other factors, such as age, the burden of individual symptoms and comorbidities is complex and highly individual.

\section{Schema therapy in ADHD: Implications for future research}

Taken together, the described cases and studies support the theory of maladaptive schemas as a relevant factor in the psychopathology of adult ADHD patients and encourage further research on schema therapy in these patients as a potentially promising approach. However, up to date no pilot studies or randomized controlled trials investigating schema therapy in ADHD patients have been conducted. The design of such studies needs to be carefully planned, particularly with regard to the outcome parameters which are expected to improve under schema therapy. Since the core symptoms of ADHD, inattention, hyperactivity and impulsivity can be seen as traits with a primarily neurobiological genesis, rather than a biographical origin, the substantial improvement of ADHD core symptoms under schema therapy seems unlikely. In contrast, the focus of schema therapy would likely be on the improvement of secondary problems, such as ineffective coping strategies and biased self-perception, resulting from the development of maladaptive schemas during childhood. In example, a "failure" schema could lead to reduced self-esteem, anxiety, social retreat and the tendency to quickly give up on difficult tasks while an emotional deprivation schema may lead to demanding behavior and instability of relationships. Such specific problems are difficult to measure and compare by validated instruments, all the more so since different patients usually have their own specific combination of maladaptive schemas and related consequences. However, consequences of maladaptive schemas can often be closely linked to common comorbidities in ADHD patients, such as depression and anxiety, which are well known from the literature ${ }^{26}$ and were confirmed in our recent study (see above) ${ }^{19}$. Thus, to obtain scientifically sound and comparable results, it might be feasible for a study on schema therapy in ADHD to focus on a subgroup of patients with a clearly defined comorbidity, such as depression, that is easily measurable and can be expected to improve under schema therapy. An alternative study approach could be the usage of more generalized outcome measurements such as the Clinical Global Impression scales (referring to a patient's overall psychiatric morbidity) or measurements of health-related quality of life and daily functioning, which may improve in patients with different maladaptive schemas by targeting their specific combination in therapy. 
A further difficulty with regard to the design of studies is the usually high individualization of schema therapy, resulting in difficulties to provide an 'equal' treatment to all participants. However, more standardized attempts on schema therapy have already been developed, such as the manual on group schema therapy for borderline personality disorder by Farrell and Shaw $^{27}$ and these may be adapted for the use in ADHD.

\section{Conclusions}

In conclusion, schema therapy or similar therapy forms, focusing on the potential burden of the patient's personal history, seem to be interesting and promising approaches in the psychotherapy of adult ADHD. Since the focus of these approaches is very different from the standard form of psychotherapy, CBT, these should be seen as an extension or addition, rather than an alternative to CBT. Particularly patients, who continue to suffer from significant secondary problems, such as depression or anxiety, under standard pharmacological and/or psychotherapeutic treatment, may profit from more biography oriented approaches such as schema therapy.

\section{Conflicts of Interest}

Caroline Lücke and Helge H. Müller declare that they have no conflicts of interest.

Alexandra Lam declares that she has received travel grants within the last year from MEDICE Arzneimittel Pütter GmbH and Co. KG.

Alexandra Philipsen has served on advisory boards, given lectures, performed phase 3 studies, or received travel grants within the last 3 years from Eli Lilly and Co, Janssen-Cilag, MEDICE Arzneimittel Pütter GmbH and Co KG, Novartis, and Shire; and has authored books and articles on psychotherapy published by Elsevier, Hogrefe, Schattauer, Kohlhammer, Karger, Springer, and Oxford Press.

\section{References}

1. Philipsen A, Hesslinger B, Tebartz van Elst L. Attention deficit hyperactivity disorder in adulthood: diagnosis, etiology and therapy. Dtsch Arztebl Int. 2008; 105(17): 311-317.

2. Association AP: Diagnostic and Statistical Manual of Mental Disorders, 5th edition. : Arlington, VA., American Psychiatric Association; 2013.

3. NICE: Attention deficit hyperactivity disorder: diagnosis and management of ADHD in children, young people and adults. National Clinical Practice Guideline Number 72. In. Edited by Excellence NIfHaC. Retrieved from https://www.nice.org.uk/guidance/cg72; 2008.

4. Wilens TE, Spencer TJ, Biederman J. A review of the pharmacotherapy of adults with attention-deficit/hyperactivity disorder. J Atten Disord. 2002; 5(4): 189-202.

5. Knouse LE, Safren SA. Current status of cognitive behavioral therapy for adult attention-deficit hyperactivity disorder. Psychiatr Clin North Am. 2010; 33(3): 497-509.

6. Ramsay J, Rostain AL. Psychosocial Treatments for Attention-Deficit/
Hyperactivity Disorder in Adults: Current Evidence and Future Directions. Professional Psychology Research and Practice. 2007; 38(4): 338-346.

7. Jensen $\mathrm{CM}$, Amdisen BL, Jorgensen KJ, et al. Cognitive behavioural therapy for ADHD in adults: systematic review and meta-analyses. Atten Defic Hyperact Disord. 2016; 8(1): 3-11.

8. Hesslinger B, Tebartz van Elst L, Nyberg E, et al. Psychotherapy of attention deficit hyperactivity disorder in adults a pilot study using a structured skills training program. Eur Arch Psychiatry Clin Neurosci. 2002; 252(4): 177-184.

9. McCullough JP Jr. Treatment for chronic depression using Cognitive Behavioral Analysis System of Psychotherapy (CBASP). J Clin Psychol. 2003; 59(8): 833-846.

10. Young JE KJ, Weishaar ME. Schema Therapy: a practitioner's guide. The Guilford Press, New York. 2003.

11. Rafaeli EB DP, Young J. Schema Therapy: Distinctive Features, CBT Distinctive Features. Routledge. 2010.

12. Renner F, Arntz A, Peeters FP, et al. Schema therapy for chronic depression: Results of a multiple single case series. J Behav Ther Exp Psychiatry. 2016; 51: 66-73.

13. Balje A, Greeven A, van Giezen A, et al. Group schema therapy versus group cognitive behavioral therapy for social anxiety disorder with comorbid avoidant personality disorder: study protocol for a randomized controlled trial. Trials. 2016; 17(1): 487.

14. Mrug S, Molina BS, Hoza B, et al. Peer rejection and friendships in children with Attention-Deficit/Hyperactivity Disorder: contributions to long-term outcomes. J Abnorm Child Psychol. 2012; 40(6): 1013-1026.

15. Ramsay JR RA. Adapting Psychotherapy to Meet the Needs of Adults With Attention-Deficit/Hyperactivity Disorder. Psychotherapy Theory Research Practice Training. 2005; 42(1): 72-84.

16. Ramsay JR RA. A cognitive therapy approach for adult attentiondeficit/hyperactivity disorder. Journal of Cognitive Psychotherapy An International Quarterly. 2003; 17: 319-334.

17. Miklosi M, Mate 0 , Somogyi $K$, et al. Adult Attention Deficit Hyperactivity Disorder Symptoms, Perceived Stress, and Well-Being: The Role of Early Maladaptive Schemata. J Nerv Ment Dis. 2016; 204(5): 364-369.

18. Kessler RC, Adler L, Ames M, et al: The World Health Organization Adult ADHD Self-Report Scale (ASRS): a short screening scale for use in the general population. Psychol Med. 2005; 35(2): 245-256.

19. Philipsen A, Lam AP, Breit S, et al. Early maladaptive schemas in adult patients with attention deficit hyperactivity disorder. Atten Defic Hyperact Disord. 2017; 9(2): 101-111.

20. Retz Junginger $P$, Retz $W$, Blocher $D$, et al. Wender Utah rating scale the short-version for the assessment of the attention-deficit hyperactivity disorder in adults. Nervenarzt. 2002; 73(9): 830-838.

21. Conners CKE D, Sparrow,EP. Conners' Adult ADHD Rating Scales (CAARS). North Tonawanda Multi Health Systems 1999.

22. Rösler MR JP, Retz W, Stieglitz RD. Homburger ADHS-Skalen für Erwachsene (HASE). Manual Göttingen Hogrefe. 2008.

23. Bakhshani NM. Attention-deficit/hyperactivity disorder (ADHD) and high risk behaviors. Int J High Risk Behav Addict. 2013; 2(1): 1-2.

24. Toplak ME, Connors L, Shuster J, et al. Review of cognitive cognitivebehavioral and neural-based interventions for Attention-Deficit/ Hyperactivity Disorder (ADHD). Clin Psychol Rev. 2008; 28(5): 801823.

25. Weusten LH, Heijnen Kohl SM, Ellison J, et al. Interference of attention-deficit hyperactivity disorder in an older adult with a severe personality disorder and dermatillomania. Int Psychogeriatr. 2014; 26(2): 341-343. 
26. Cumyn L, French L, Hechtman L. Comorbidity in adults with attentiondeficit hyperactivity disorder. Can J Psychiatry. 2009; 54(10): 673683.
27. Farrell JMS IA. Group Schema Therapy for Borderline Personality Disorder: A Step-by-Step Treatment Manual with Patient Workbook. Wiley-Blackwell. 2012. 